\title{
A role of TTI1 in the colorectal cancer by promoting proliferation
}

\author{
Peng Xu, Guangsheng Du, Haidi Guan, Weidong Xiao, Lihua Sun, Hua Yang \\ Department of General Surgery, the Second Affiliated Hospital, Army Medical University, Chongqing, China \\ Contributions: (I) Conception and design: P Xu; (II) Administrative support: H Yang, L Sun; (III) Provision of study materials or patients: G Du; \\ (IV) Collection and assembly of data: P Xu; (V) Data analysis and interpretation: P Xu; (VI) Manuscript writing: All authors; (VII) Final approval of \\ manuscript: All authors. \\ Correspondence to: Lihua Sun, MS; Hua Yang, MD, PhD. Department of General Surgery, the Second Affiliated Hospital, Army Medical University, \\ Chongqing 400037, China. Email: slh6260@163.com; hwbyang@126.com.
}

Background: Colorectal cancer (CRC) is one of the most malignant cancer worldwide, which leads to a
high incidence and mortality. The molecular mechanism in CRC is still limited. The aim of this study was to
identify hub genes and its related function in CRC.

Methods: The expression dataset (GSE44076) was downloaded from Gene Expression Omnibus (GEO) and differentially expressed genes (DEGs) analysis was done using R 'limma' packages. Weighted gene co-expression network analysis (WGCNA) was done and tumor-specific modules were picked up for Gene Ontology (GO) and Kyoto Encyclopedia of Genes and Genomes (KEGG) analysis. The hub gene was selected with higher inter-connectivity. Expression levels of TTI1 were verified by in clinical CRC tissues. The cell counting kit-8 (CCK-8) assay was to measure the proliferative ability of TTI1.

Results: Eight hundred and eight up-regulated and 929 down-regulated DEGs were screened out. Up-regulated genes enriched in cell proliferation and down-regulated genes enriched in oxidation-reduction process. After WGCNA, the yellow module was found to be the most significant tumor-specific module. Function analysis showed genes in the yellow module enriched in oxidation-reduction, cell proliferation and extracellular matrix (ECM)-receptor interaction. TTI1 was demonstrated as the hub gene. Real-time quantitative reverse transcription (qRT-PCR) results showed TTI1 significantly expressed higher in CRC tissues than adjacent normal tissues. TTI1 dramatically correlated with proliferation in CRC.

Conclusions: These findings regarded TTI1 as a vital promoting factor in CRC development and provided a potential biomarker for CRC treatment.

Keywords: TTI1; colorectal cancer (CRC); weighted gene co-expression network analysis (WGCNA); proliferation

Submitted Nov 25, 2020. Accepted for publication Jan 22, 2021.

doi: $10.21037 /$ tcr-20-3322

View this article at: http://dx.doi.org/10.21037/tcr-20-3322

\section{Introduction}

Colorectal cancer (CRC) is an important contributor to new cancer cases each year (1). In China, the mortality of CRC is 7.8 per 100,000 in 2011 and is forecasted to reach 8.6 per 100,000 in 2020 (2). CRC also causes the second highest deaths in the United States in 2020 (3). Hence, an effective therapy for CRC is of prime importance. With the development of the biotechnology, epigenetic alterations are providing potential biomarkers for diagnosis, therapeutic plans and prognosis of the CRC (4). On the basis of microarray technology and bio-informatics analysis, gene expression analysis is drawing more attention to the molecular field and helps us discover differentially expressed genes (DEGs) in CRC $(5,6)$. However, transcription analysis presents hundreds of DEGs, raising the level of challenges and complexity to search the target gene. Moreover, co-expression clustering is a conventional method to extract genetic interactions and highlight common features of DEGs in CRC $(7,8)$. Weighted gene co-expression network 
analysis (WGCNA) is regarded as a systematic biological strategy to screen out modules with higher genetic correlations (9). By identifying gene modules and relative phenotypes, we can study gene modules with significant synergistic action and find hub genes in interesting modules.

In this study, the gene expression profiles of CRC tissues and adjacent normal tissues by microarray analysis were downloaded from Gene Expression Omnibus (GEO) and screened out DEGs $(10,11)$. Then WGCNA was applied to divide DEGs into multiple co-expression modules and screened out tumor-specific modules. Kyoto Encyclopedia of Genes and Genomes (KEGG) enrichment analysis were performed and provide insight into metabolic processes and functions of gene products (12). Finally, a gene network was set up to identify hub genes upon inter-modular connectivity $(13,14)$. Ultimately, the differentially expressed level and the biological tumor-specific function of the hub gene were verified. The function and clinical role were then performed. Taken as a whole, the hub gene may play a critical role in the occurrence and development of the CRC and provide a potential target for CRC treatment strategy. We present the following article in accordance with the MDAR reporting checklist (available at http://dx.doi. org/10.21037/tcr-20-3322).

\section{Methods}

\section{Ethical statement}

Our work was approved by the ethics committee of the Army Medical University. All experiments were completed in specific pathogen-free conditions. The study was conducted in accordance with the Declaration of Helsinki (as revised in 2013). The study was approved by the Ethics Committee of the Army Medical University (ChiCTR2000033078) and individual consent for this retrospective analysis was waived.

\section{Microarray data source and analysis}

The gene expression dataset (GSE44076) was downloaded from GEO. Gene expression profiles of paired normal adjacent mucosa and tumor samples from 98 individuals and 50 healthy colon mucosae were obtained through Affymetrix Human Genome U219 Arrays. DEGs were obtained through $\mathrm{R}$ language software 3.6.1 using 'limma' packages. Gene symbols showed non-correspondence with probes or multiple probes corresponding to the same gene were moved out or taken the average. We recommended that $\mid \log$ (fold change) $\mid(|\log \mathrm{FC}|)>1, \mathrm{P}$ value $<0.05$ is statistically significant.

\section{WGCNA construction}

The DEGs were filtered out and performed WGCNA analysis through R "WGCNA" packages. To reduce the computational burden without losing important information, $\mid \log \mathrm{FCl}>0.585$ and $\mathrm{P}<0.05$ were taken as the standard for mRNA-choosing. In order to stabilize the result, we picked up a soft threshold power to construct the co-expression modules. The average value of connective degree was calculated. A hierarchical clustering tree was built after the matrix conversion in line with the soft threshold and genetic expression data. The colorful cluster dendrograms of tumor and normal tissues were constructed and we took the minModuleSize as 30 in each dendrogram. The associations between the tumor set-specific module and the tumornormal consensus module were defined using R "WGCNA" packages. A deeper color stands for a closer correlation. Tumor-specific modules with a significant contrast with normal modules were chosen for further analysis.

\section{Gene Ontology (GO) and KEGG analysis}

David (http://david.ncifcrg.gov) (version 6.8), the abbreviation of "the Database for Annotation, Visualization and Integrated Discovery", provides series tools for functional annotation and biological meaning of genes. In our research, we used 'David' database to analyze biological processes (BPs), cellular components (CCs), molecular functions (MFs) and enrichment of signaling pathways of genes in hub modules. $\mathrm{P}$ value $<0.05$ was to be statistically significant.

\section{Hub gene identification}

Total 3,864 genes of the tumor-specific module were detected and the internal connections among those genes were calculated. We chose top 30 genes to be visualized by Cytoscape 3.7.2. The top 5 genes with highest connectivity within the module were defined as hub genes.

\section{Database analysis}

GEPIA, the abbreviation of 'Gene Expression Profiling 
Interactive Analysis', is an online database providing RNAseq data of TCGA and GTEx database. We verified the differential expression of hub genes based on the GEPIA and showed in boxplots. The expression data of TTI1, Ki-67 and PCNA were from GEO database. The survival data of CRC patients was downloaded from TCGA database and analyzed by the $\mathrm{R}$ 'survival' package and 'survminer' package. TTI1 expression data of CRC patients with clinical traits were downloaded from USCS Xena (https://xena.ucsc.edu). The PPI network and function of the hub gene were obtained from String (https://www. string-db.org/).

\section{Colorectal tissues collection}

Our work was approved by the Ethics Committee of the Army Medical University and the experimental protocols were accorded with the Declaration of Helsinki (clinical trial register no. ChiCTR2000033078). The clinical tissues were used for the scientific investigation with the permissions of all the patients. Patients were selected according to the admission time (from 2017 to 2019) in the Second Affiliated Hospital of the Army Medical University. Patients were diagnosed pathologically and 53 pairs of colorectal tissues and adjacent normal tissues were removed after operation and rapidly stored at $-80^{\circ} \mathrm{C}$.

\section{Cell culture and transfection}

Human CRC cancer cell lines $\left(\mathrm{CaCO}_{2}, \mathrm{HT} 29\right)$ were purchased from the Type Culture Collection of Chinese Academy of Sciences (Shanghai, China). $\mathrm{CaCO}_{2}$ cells were maintained in complete MEM medium with $10 \%$ bovine serum albumin, $1 \%$ penicillin-streptomycin solution and 1\% nonessential amino acid solution. HT29 cells were maintained in McCoy's 5a complete medium with $10 \%$ bovine serum albumin and $1 \%$ penicillinstreptomycin solution. Cells were incubated at $37{ }^{\circ} \mathrm{C}$ with $5 \% \mathrm{CO}_{2}$ and $20 \% \mathrm{O}_{2}$. To reduce TTI1 expression, small interfering RNA (siRNA) was used coupled with Lipofectamine $^{\mathrm{TM}} 2000$ Transfection Reagent (Invitrogen, USA). siRNA duplexes targeting the TTI1 mRNA were synthesized by Ribobio (Guangzhou, China) as following: GGAAGCAAGTGTGGTGACT. The density of cells reached $30-50 \%$ and then cells were transfected with the siRNA targeting TTI1 and negative control siRNA (siCon) through the use of Lipofectamine ${ }^{\mathrm{TM}} 2000$ Transfection Reagent (Invitrogen, USA). After a 6-hour transfection, cells were purified for proliferation assay.

\section{Real-time quantitative reverse transcription (qRT-PCR) detecting system}

Total RNA was isolated from clinical tissues collected in the Second Affiliated Hospital of the Army Medical University using TRIzol (Invitrogen, Carlsbad, CA, USA) in line with the manufacturers' instructions. The concentration determination of RNA was based on NanoDrop 2000 (Thermo Fisher Scientific, MA, USA). A SuperScript First-Strand Synthesis System RT-PCR kit (TaKaRa Bio Inc., Japan) was used to reverse-transcribe RNA into complementary DNA (cDNA). GoTAq qPCR Master Mix (Promega, USA) was participated into the PCR process based on the Rotor-Gene Q PCR detection system (Qiagen, Germany). Sequences of primers are as following: TTI1 forward, 5'-CTGGCGACAAGTTGACTATCCT-3'; TTI1 reverse, 5'-CCAGTTCCAGTCTCACCTTCCA-3'; GAPDH forward: 5'-AGCCACATCGC TCAGACAC-3'; GAPDH reverse: 5'-GCCCAATACGACCAAATCC-3'. The PCR process was performed in a $10 \mu \mathrm{L}$ system with $95^{\circ} \mathrm{C}$ for $10 \mathrm{~min}, 40$ cycles of $95^{\circ} \mathrm{C}$ for $5 \mathrm{~s}$ and $60{ }^{\circ} \mathrm{C}$ for $30 \mathrm{~s}$ and $72{ }^{\circ} \mathrm{C}$ for $30 \mathrm{~s}$. The expression data was calculated by $2^{-\Delta \Delta \mathrm{T}}$.

\section{Western blot}

Proteins of clinical samples from patients were extracted upon RIPA and PMSF (BOSTER, USA). After polyacrylamide gel transfer electrophoresis, samples were transferred to a PVDF (polyvinylidene difluoride) membrane. The membrane was blocked and incubated with the primary antibody against TTI1 (SANTA CRUZ, sc-365119, 1:100) overnight. Then the membrane was incubated with secondary antibody for 1 hour. Results were detected using Western Blot Imaging System (4000R, Care Tream, USA).

\section{Immunofluorescence}

Four percent paraformaldehyde (Boster Biological Technology) was used to fix samples and the primary antibody against TTI1 (SANTA CRUZ, sc-365119, 1:50) was incubated with samples at $4{ }^{\circ} \mathrm{C}$ overnight. Then the secondary antibody (Abcam, ab150113, 1:500) and DAPI (Beyotime Biotechnology) was used separately to stain samples. A TCS-SP5 confocal microscope (Leica, Germany) was used for visualization. 
A

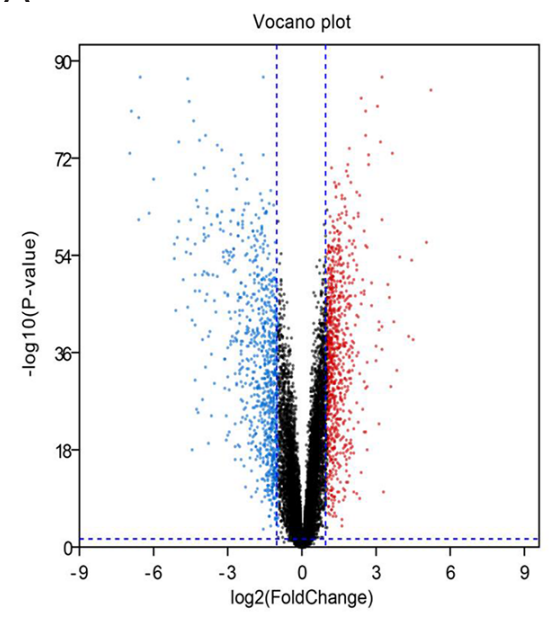

D

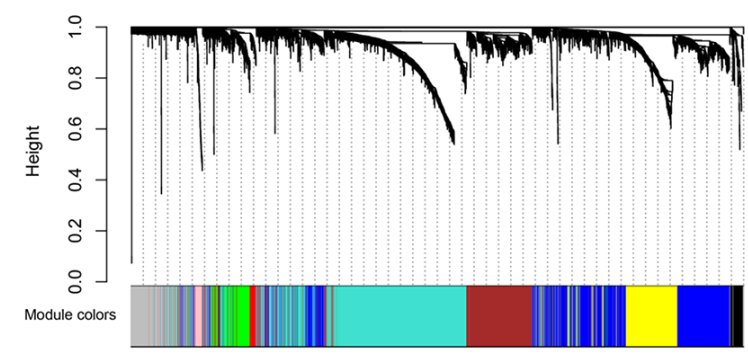

B

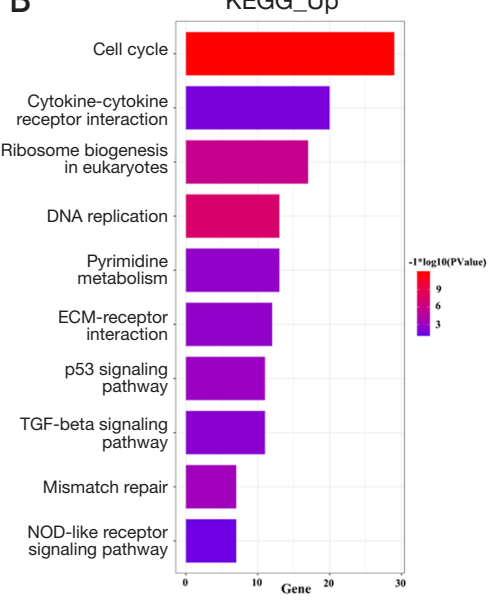

$E$

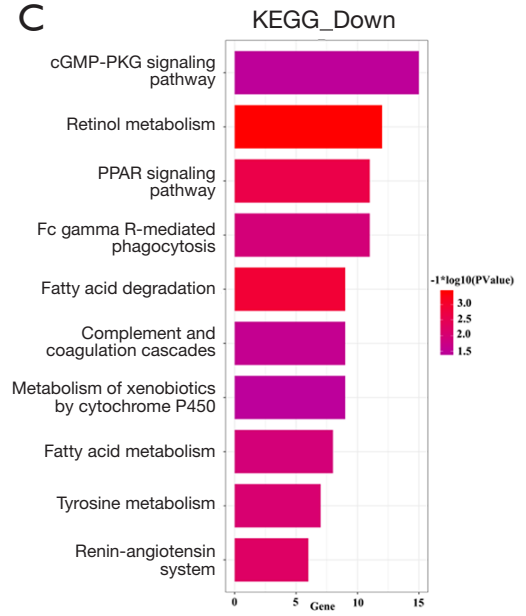

Cluster dendrogram for tumor

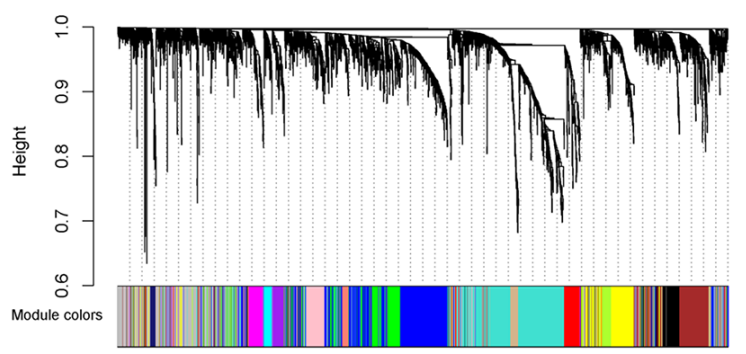

Figure 1 DEGs identified in GSE44076. (A) Volcano plot DEGs in GSE44076. Red color: up-regulated in GSE44076. Blue color: downregulated in GSE44076. Black color: no difference genes. (B) Top 10 KEGG pathway of up-regulated DEGs. (C) Top 10 KEGG pathway of down-regulated DEGs. (D) Cluster dendrogram of normal mucosa. (E) Cluster dendrogram of tumor. DEG, differentially expressed gene; KEGG, Kyoto Encyclopedia of Genes and Genomes; ECM, extracellular matrix.

\section{Cell proliferation assay}

Cell counting kit-8 (CCK-8; Dojindo, Shanghai, China) was used to demonstrate the proliferative ability of $\mathrm{CaCO}_{2}$ and HT29 cells. Cells were cultured in 96-well plates and each well contained $3 \times 10^{3} /$ cells. Twenty-four hrs after transfection, $200 \mu \mathrm{L}$ CCK-8 solution was added into wells and the optical density was qualified after $2 \mathrm{~h}$ cell-incubating at $37^{\circ} \mathrm{C}$. The absorbance was measured by the Multiskan spectrum (Berthod, Germany) at $450 \mathrm{~nm}$. Similarly, the optical density was measured in the following 7 days.

\section{Statistical analysis}

Data was expressed as the mean \pm SD. Statistic analysis was done using Graphpad Prism 7.0 (San Diego, CA, USA). Pearson's correlation coefficients and the two-tailed Student $t$-test determined the statistical significance. $\mathrm{P}<0.05$ is statistically significant.

\section{Results}

\section{DEGs and constructing weighed co-expression network}

Ninety-eight CRC samples and 50 normal tissue samples were included in the gene expression profile (GSE44076) in this study. Correction data was assigned to DEG analysis and 1,727 DEGs (808 up-regulated and 929 downregulated) were screened out according to the condition of $\mid \log \mathrm{FCl}>1, \mathrm{P}<0.05$ (Figure 1A). GO and KEGG analysis were then performed using 'David'. Up-regulated genes were enriched in cell proliferation, DNA-templated and proteinaceous extracellular matrix (ECM), however, downregulated genes were enriched in oxidation-reduction process, inflammatory response and cell surface in GO analysis (Figure S1A,B). KEGG analysis showed that up- 
A

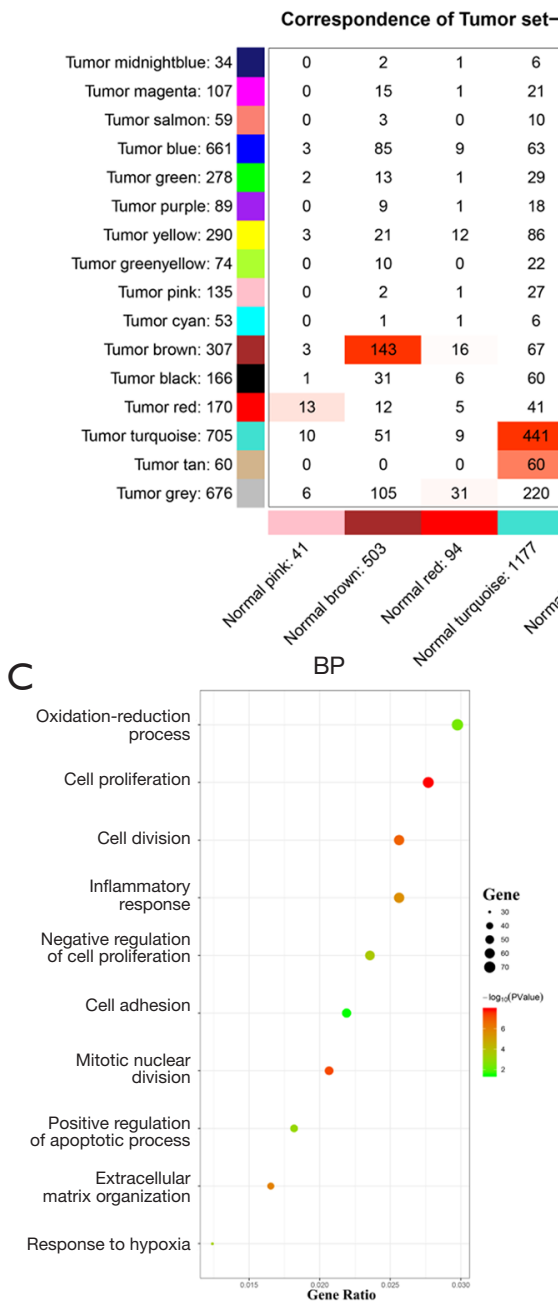

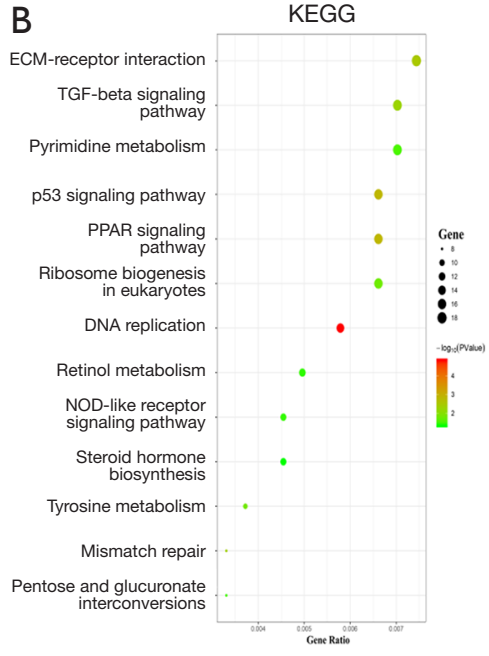

$\mathrm{CC}$
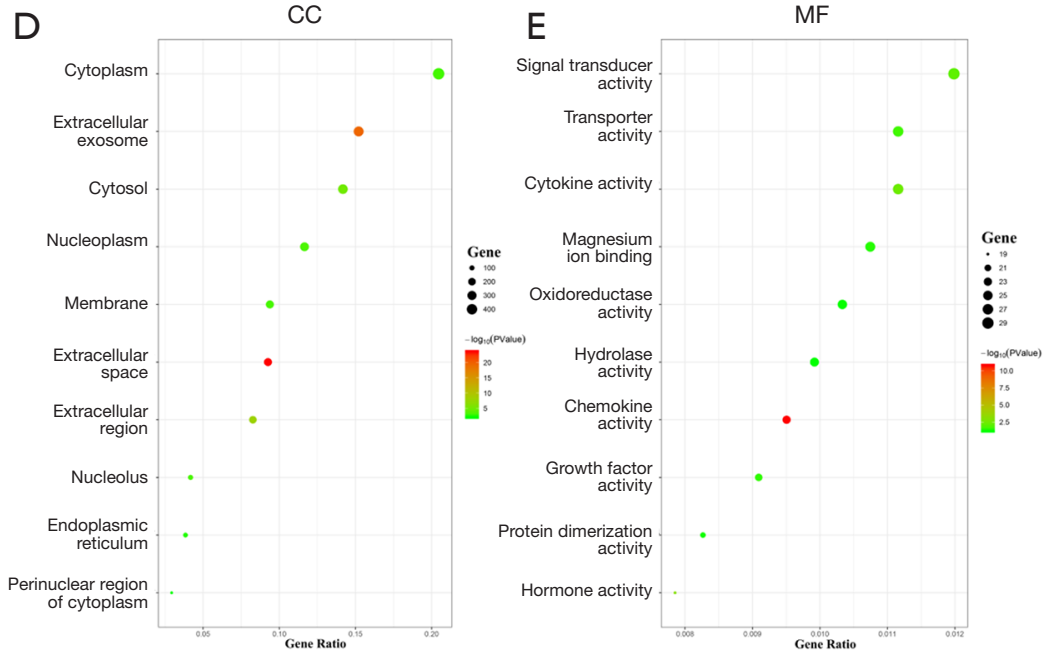

Figure 2 Identifications and function analysis of the hub module. (A) Heatmap of the correlation between Tumor set-specific modules and normal modules. (B) Top 13 KEGG pathways of genes in the yellow module. (C) Top 10 BP terms of genes in the yellow module. (D) Top $10 \mathrm{CC}$ terms of genes in the yellow module. (E) Top $10 \mathrm{MF}$ terms of genes in the yellow module. KEGG, Kyoto Encyclopedia of Genes and Genomes; BP, biological process; CC, cell component; MF, molecular function; ECM, extracellular matrix.

regulated genes were enriched in cell cycles, cytokinecytokine receptor interaction, ribosome biogenesis and down-regulated genes were enriched in cGMP-PKG signaling pathway, retinol metabolism and PPAR signaling pathway (Figure 1B,C). To provide a further insight into the correlations among DEGs, the WGCNA analysis was performed using R 'WGCNA' packages. Four normal samples (GSM1077708, GSM1077694, GSM1077670 and GSM1077712) and one tumor sample (GSM1077800) were discarded after sample clustering (Figure S1C,D). The soft threshold power was separately identified as 6 and $5\left(\mathrm{R}^{2}>0.9\right)$ of normal and tumor samples (Figure S1E,F).
The hierarchical cluster analysis was conducted and gene modules were obviously showed (Figure 1D,E).

\section{Identification of the bub module and function enrichment analysis}

To investigate the gene expression difference between tumor and normal tissues, we compared gene modules of tumor with those of normal tissues. The results showed the correlation between tumor set-specific modules and tumornormal consensus modules (Figure 2A). Modules of tumor samples with the lowest correlation and the biggest difference 
A

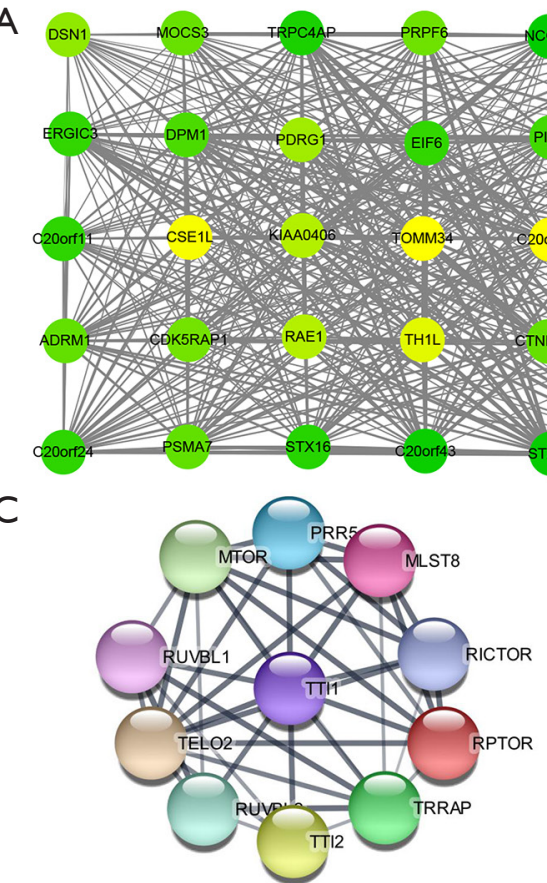

B

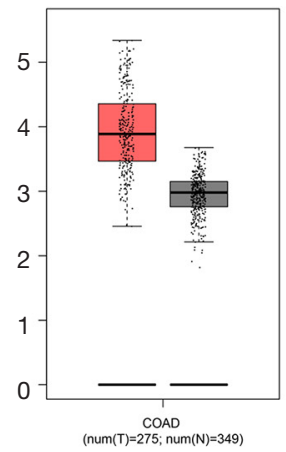

D

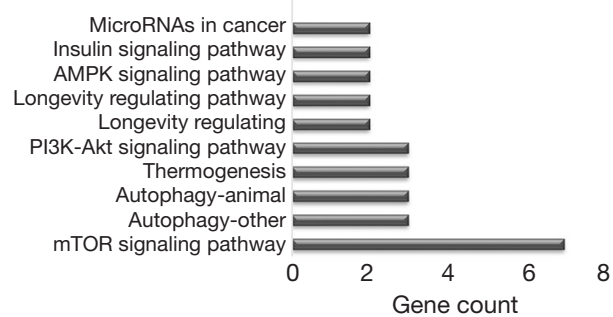

Figure 3 Identification of the hub gene and the relative function. (A) The top 30 hub genes in the yellow module. Nodes are correlated with inter-modular connectivity by degree and the gradual color change from yellow to green represents logFC. (B) The mRNA level of TTI1 in COAD patients from GEPIA. (C) PPI network of TTI1 from String database. (D) Function analysis of TTI1. FC, fold change; COAD, colon adenocarcinoma; GEPIA, Gene Expression Profiling Interactive Analysis; PPI, protein-protein interaction.

against modules of normal tissues were identified as tumorspecific modules. Results demonstrated that the yellow module was the most tumor-specific module. Therefore, the yellow module was identified as the hub module and suggested to play a critical role in the CRC. To classify the biological function of genes in the hub module, the GO process and the KEGG analysis were performed. The KEGG analysis demonstrated that genes of the hub module were enriched in ECM-receptor interaction, TGF-beta signaling pathway and pyrimidine metabolism (Figure 2B). Results of GO analysis discovered that genes were focused on the oxidation-reduction, cell proliferation and cell division on BP (Figure 2C). Moreover, genes were enriched in cytoplasm, extracellular exosome and cytosol on CC (Figure 2D). In MF analysis, signal transducer activity, transporter activity and cytokine activity were significant for genetic enrichment (Figure 2E).

\section{Identification of hub genes and verifying expression levels}

To identify hub genes in the yellow module, genes were ranked according to the inter-modular connectivity. The top
30 genes with the highest inter-modular connectivity were chosen for visualization using Cytoscape3.7.2 (Figure 3A). The bigger size of nodes stood for a higher inter-modular connectivity and the color of nodes stood for the FC. Finally, the top five genes with the highest inter-connectivity (STAU1, KIAA0406, DPM1, CTNNBL1, C20orf11/ GID8) were identified as hub genes. Then expression levels of hub genes were identified in the GEPIA database. All hub genes except DPM1, showed a significant difference of the expression level (Figure 3B, Figure S2A). However, KIAA0604 (also named TTI1) showed the most significant differentially expressed level and the second highest connectivity among five hub genes. Therefore, we chose TTI1 for a further analysis. Protein-protein network (PPI) of TTI1 was performed and visualized (Figure 3C). Function analysis using String database was also proved that TTI1 was associated with PI3K/AKT/mTOR pathway (Figure 3D).

\section{TTI1 is up-regulated in CRC}

The above part showed the TTI1 as a hub gene with differential expression in CRC tissues rather than normal 
A

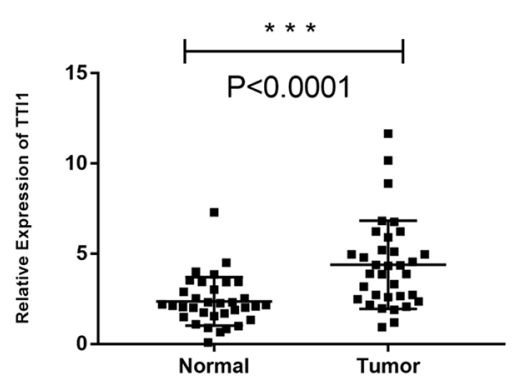

B

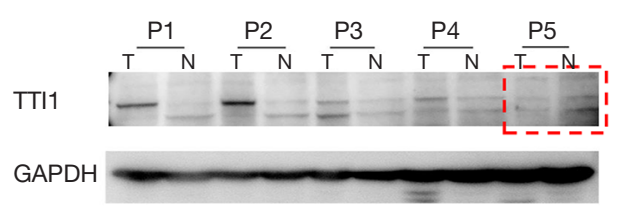

C

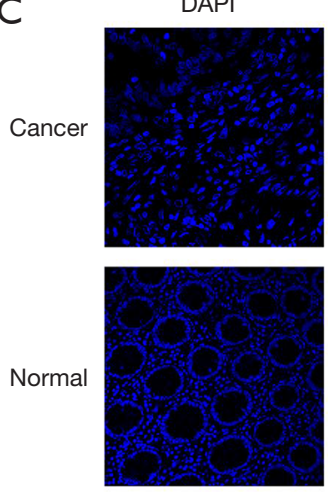

D

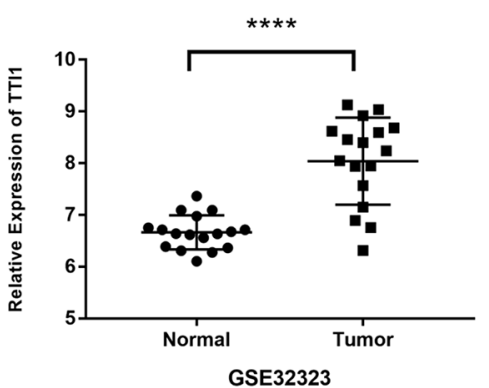

TTI1
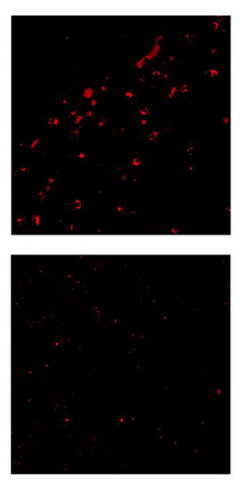

E

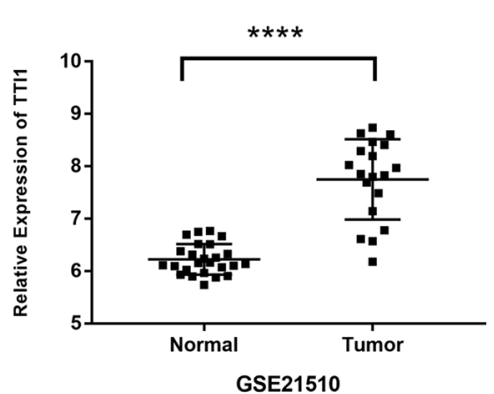

Figure 4 TTI1 is overexpressed in CRC. (A) TTI1 expression in CRC tissues vs. adjacent normal mucosa at the mRNA level ${ }^{* * *}$, $\mathrm{P}<0.0001$ ). (B) TTI1 expression in CRC tissues $v s$. adjacent normal mucosa at the protein level. (C) Immunofluorescence showing TTI1 expression in CRC tissues vs. adjacent normal mucosa. (The magnification is 1,200 times, arrows mark where the TTI1 is expressed). (D) TTI1 expression in GSE32323 and GSE21510 (****, P<0.001). CRC, colorectal cancer.

tissues. We then demonstrated the expression level of clinical CRC tissues and paired adjacent tissues collected from patients in the Second Affiliated Hospital of the Army Medical University. qRT-PCR and Western Blot verified that TTI1 was significantly up-regulated in CRC tissues than adjacent tissues (Figure 4A,B). Immunofluorescence also demonstrated that TTI1 was highly expressed in colorectal tissues compared with adjacent normal tissues (Figure 4C). The result screened out that TTI1 showed higher expression in tumor tissues. However, clinical factors [age, gender, differentiated degree, stage, body mass index (BMI) and occurrence site of cancer] showed no significance between groups with lower and higher expression of TTI1 (Table 1). Moreover, expression data of GSE32323 and GSE21510 revealed that TTI1 was significantly up-regulated in CRC tissues compared with normal samples (Figure 4D).

\section{TTI1 is involved in the proliferation of CRC}

The survival curves with different TTI1 expression were performed and there was no significant correlation between survival time and the expression of TTI1 (Figure S2B,C). Moreover, analysis using USCS Xena database demonstrated that a higher level of TTI1 showed no significant correlation with the lymphatic invasion and the venous invasion (Figure S2D,E). Unregulated cell proliferation is closely to tumor progression in the early stage of CRC. Correlation analysis in GSE32323 and GSE21510 identified that there was a positive correlation between the expression level of TTI1 with expression levels of proliferation-related markers Ki-67 and PCNA (Figure $5 A, B, C, D$ ). We then probed into the function of TTI1 in CRC cell lines. The HT29 cells and $\mathrm{CaCO}_{2}$ cells were transfected with TTI1 siRNA. Moreover, the proliferative ability of transfected cells was decreased significantly (Figure 5E,F). Therefore, TTI1 played a positive role in promoting cell proliferation of CRC.

\section{Discussion}

CRC as a modern disease, is increasingly common while 
Table 1 The correlation between TTI1 expressed levels and clinicopathological characteristic in CRC (53 pairs)

\begin{tabular}{|c|c|c|c|c|}
\hline \multirow{2}{*}{ Characteristics } & \multicolumn{2}{|c|}{ TTI1 expression } & \multirow{2}{*}{ Total } & \multirow{2}{*}{$P$ value } \\
\hline & High & Low & & \\
\hline Age & & & & 0.26 \\
\hline$>60$ & 23 & 9 & 32 & \\
\hline$\leq 60$ & 12 & 9 & 21 & \\
\hline Gender & & & & 0.56 \\
\hline Male & 26 & 12 & 38 & \\
\hline Female & 9 & 6 & 15 & \\
\hline Differentiated degree & & & & 0.68 \\
\hline Poorly differentiated & 14 & 9 & 23 & \\
\hline Moderately differentiated & 18 & 7 & 25 & \\
\hline Well differentiated & 3 & 2 & 5 & \\
\hline Stage & & & & 0.42 \\
\hline Stage I & 7 & 3 & 10 & \\
\hline Stage II & 14 & 9 & 23 & \\
\hline Stage III & 14 & 5 & 19 & \\
\hline Stage IV & 0 & 1 & 1 & \\
\hline $\mathrm{BMI}$ & & & & 0.48 \\
\hline$>24$ & 11 & 4 & 15 & \\
\hline$\leq 24$ & 24 & 14 & 38 & \\
\hline Occurrence site & & & & 0.48 \\
\hline Ascending colon & 5 & 5 & 10 & \\
\hline Transverse colon & 1 & 0 & 1 & \\
\hline Transverse colon & 2 & 0 & 2 & \\
\hline Sigmoid colon & 8 & 7 & 15 & \\
\hline Rectum & 19 & 6 & 25 & \\
\hline
\end{tabular}

$\mathrm{CRC}$, colorectal cancer; BMI, body mass index.

the mode of life is shifting to the Western style. Risk factors (smoking, obesity and alcoholism) make the disease get out of control. The incidence of young people is increasing (15). Therefore, it is necessary to improve an effective method of diagnosis and treatment. In the past decades, screening CRC using colonoscopy enhanced detection and reduced incidence of the cancer, but the survival fate is still poor $(16,17)$. Genes related to prognosis are increasingly discovered in recent years (18). Nonetheless, targeted therapies for CRC patients is still limited (19). New drugs for immunological therapy show less obvious effects (20). Therefore, a new biomarker as a target of diagnosis or therapies for CRC may be a meaningful direction for scientific researches.

In this study, we compared tissues of the primary colorectal adenocarcinoma and matched distant colon mucosae using RNA-seq. The expression data files and matched clinical data were downloaded from GEO and DEGs were screened out using R 'limma' packages. Eight hundred and eight up-regulated genes were mainly enriched in cell proliferation, however, 929 down-regulated genes were enriched in oxidation-reduction process and inflammatory response on basis of 'David' database. Then a deeper research was done to explore biological expectations of co-expression genes using R 'WGCNA' packages. Then a tumor-specific module (the yellow module) was screened out with the most significant difference. Genes in the yellow module were focused on biological pathways of cell proliferation according to 'David' online database. To narrow the range and find hub genes in the module, 'Cytoscape' was used to build a WGCNA network based on the inter-connectivity and the FC of genes in this module. Genes with the top five highest inter-connectivity were screened out as hub genes (STAU1, KIAA0406, DPM1, CTNNBL1, C20Orf11). KIAA0406 is also called TTI1 and C20orf11 has another name called GID8. Expression levels were verified from GEPIA database (21). Except for DPM1, other four hub genes showed a significant difference. Moreover, TTI1, with the second highest connectivity and the highest differential expressing level, was highly specific in 53 CRC samples collected in the Second Affiliated Hospital of the Army Medical University and in GEO datasets (GSE32323 and GSE21510).

TTI1 participates in constituting and stabilizing proteins of PIKK family including mTOR, ATR, DNA-PKcs and ATM as a co-chaperon $(22,23)$. TTI1 also participated in maturating PIKKs members acting with Hsp90 (24). PIKKs play a vital regulator in biological pathways of cell growth, proliferation, translation and autograph $(25,26)$. PIKK family members (ATM, ATR, DNA-PKcs, mTOR) played core roles in regulating cell proliferation in cancers $(27,28)$. However, TTI1 as a key regulator in many biologic processes, few studies were done correlated with TTI1. TTI1 is involved in multiple metabolic pathways and mTORC1 signaling activation which promotes cell growth (29). In Kristen's study, TTI1 participated in DNA damage response and was required to establish the intra-S and G2/M checkpoints (30). Previous study showed TTI1 participated in promoting survival in multiple myeloma 
A

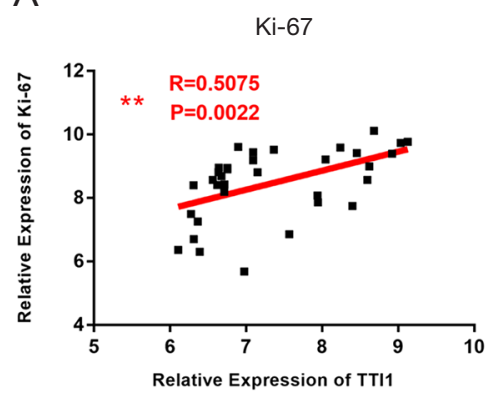

B

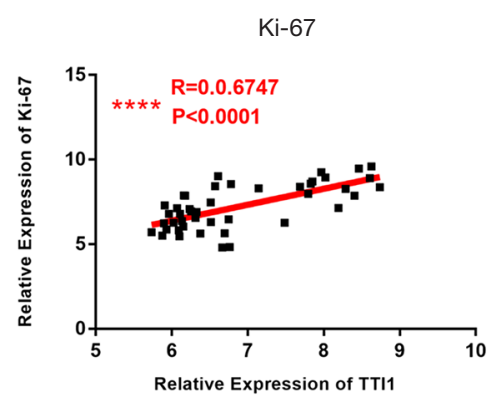

C

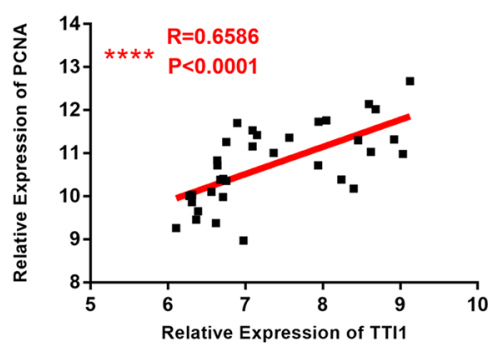

PCNA

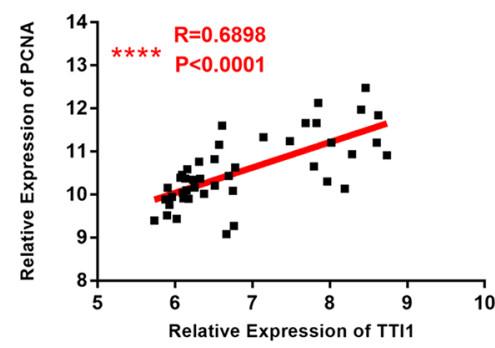

E HT29

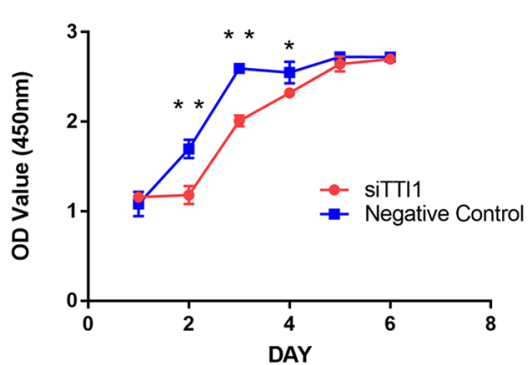

$\mathrm{F}$

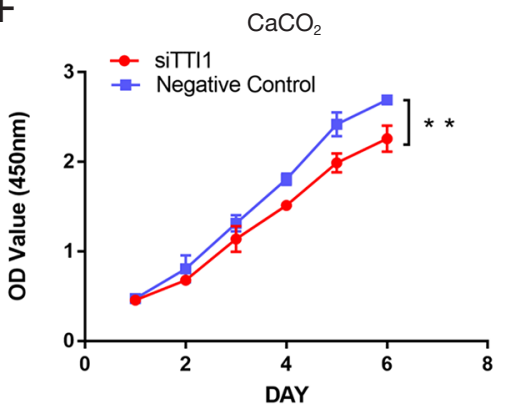

Figure 5 TTI1 promotes proliferation in CRC. (A) Correlation between TTI1 and Ki-67 in GSE32323 (**, P<0.01). (B) Correlation between TTI1 and Ki-67 in GSE21510 (****, P<0.001). (C) Correlation between TTI1 and PCNA in GSE32323 (****, P<0.001). (D) Correlation between TTI1 and PCNA in GSE21510 (****, P<0.001). (E) The absorbance value at 450 nm of HT29 cell lines transfected with TTI1 (siTTI1) and negative control (siCon) (*, $\mathrm{P}<0.1$; ${ }^{* *}, \mathrm{P}<0.01$ ). (F) The absorbance value at $450 \mathrm{~nm}^{\circ} \mathrm{CaCO}_{2}$ cell lines transfected with TTI1 (siTTI1) and negative control (siCon) $\left.{ }^{* *}, \mathrm{P}<0.01\right)$. CRC, colorectal cancer.

through mTORC1 pathway and was significantly altered in number of bladder cancer $(31,32)$. Rao et al. demonstrated that TTI1 participated in binding DNA-PKcs and ATM, which activated phosphorylation of S-15 and p-53 pathway, activated the cell death program of cancer cells $(33,34)$.

Nevertheless, the role of TTI1 in the carcinogenesis and development of the CRC is still uncleared. In this study, we screened out a higher expression level of TTI1 using bio-informatics methods and proved that TTI1 was significantly up-regulated in CRC samples collected in the Second Affiliated Hospital of the Army Medical University. However, CRC patients with higher expression levels of TTI1 were not proved to have a worse prognosis including survival time and lymphatic/venous invasion. Our function analysis showed TTI1 may participate in cell proliferation. Correlation analysis identified that the TTI1 showed a positive correlation with proliferative markers (Ki-67 and PCNA) significantly $(35,36)$. Cell proliferation assay showed down-regulated TTI1 significantly suppressed proliferation of CRC cells. It's universally accepted that uninhibited cell proliferation is the most fundamental trait and malignant hallmark of cancers (37). Hence, silence of TTI1 may significantly inhibit the early process of the CRC development. Nevertheless, targeting proliferation of cancer cells may only aim at cells with rapid proliferation. Inhibitory effect on cancer stem cells with slower proliferation requires for a further analysis $(38,39)$.

In conclusion, TTI1 expressed specially in tumor tissues than adjacent normal tissues of CRC patients and was a key factor in the proliferative process of CRC. TTI1 may play a vital bio-marker in anti-tumor researches and treatment of CRC.

\section{Acknowledgments}

Funding: This work was supported by the National Natural Science Foundation of China (Grant No. NSFC 81873551, NSFC 81873933).

\section{Footnote}

Reporting Checklist: The authors have completed the MDAR 
reporting checklist. Available at http://dx.doi.org/10.21037/ tcr-20-3322

Peer Review File: Available at http://dx.doi.org/10.21037/tcr20-3322

Conflicts of Interest: All authors have completed the ICMJE uniform disclosure form (available at http://dx.doi. org/10.21037/tcr-20-3322). The authors have no conflicts of interest to declare.

Ethical Statement: The authors are accountable for all aspects of the work in ensuring that questions related to the accuracy or integrity of any part of the work are appropriately investigated and resolved. The study was conducted in accordance with the Declaration of Helsinki (as revised in 2013). The study was approved by the Ethics Committee of the Army Medical University (ChiCTR2000033078) and individual consent for this retrospective analysis was waived.

Open Access Statement: This is an Open Access article distributed in accordance with the Creative Commons Attribution-NonCommercial-NoDerivs 4.0 International License (CC BY-NC-ND 4.0), which permits the noncommercial replication and distribution of the article with the strict proviso that no changes or edits are made and the original work is properly cited (including links to both the formal publication through the relevant DOI and the license). See: https://creativecommons.org/licenses/by-nc-nd/4.0/.

\section{References}

1. Schreuders EH, Ruco A, Rabeneck L, et al. Colorectal cancer screening: a global overview of existing programmes. Gut 2015;64:1637-49.

2. Zhu J, Tan Z, Hollis-Hansen K, et al. Epidemiological trends in colorectal cancer in China: an ecological study. Dig Dis Sci 2017;62:235-43.

3. Siegel RL, Miller KD, Jemal A. Cancer statistics, 2020. CA Cancer J Clin 2020;70:7-30.

4. Okugawa Y, Grady WM, Goel A. Epigenetic alterations in colorectal cancer: emerging biomarkers. Gastroenterology 2015;149:1204-25.e12.

5. Zhang S, Cao J. A close examination of double filtering with fold change and $t$ test in microarray analysis. BMC Bioinformatics 2009;10:402.

6. Yang $\mathrm{H}$, Lin $\mathrm{H}$, Liu H, et al. A 6 lncRNA-based risk score system for predicting the recurrence of colon adenocarcinoma patients. Front Oncol 2020;10:81.

7. Abu-Jamous B, Kelly S. Clust: automatic extraction of optimal co-expressed gene clusters from gene expression data. Genome Biol 2018;19:172.

8. Zhang X, Bai J, Yuan C, et al. Bioinformatics analysis and identification of potential genes related to pathogenesis of cervical intraepithelial neoplasia. J Cancer 2020;11:2150-7.

9. Langfelder P, Horvath S. WGCNA: an R package for weighted correlation network analysis. BMC Bioinformatics 2008;9:559.

10. Ritchie ME, Phipson B, Wu D, et al. limma powers differential expression analyses for RNA-sequencing and microarray studies. Nucleic Acids Res 2015;43:e47.

11. Barrett T, Wilhite SE, Ledoux P, et al. NCBI GEO: archive for functional genomics data sets--update. Nucleic Acids Res 2013;41:D991-5.

12. Chen $R, G e T$, Jiang $W$, et al. Identification of biomarkers correlated with hypertrophic cardiomyopathy with coexpression analysis. J Cell Physiol 2019;234:21999-2008.

13. Su G, Morris JH, Demchak B, et al. Biological network exploration with Cytoscape 3. Curr Protoc Bioinformatics 2014;47:8.13.1-24.

14. Yu G, Wang L, Han Y, et al. clusterProfiler: an R package for comparing biological themes among gene clusters. OMICS 2012;16:284-87.

15. Li GM, Zhang CL, Rui RP, et al. Bioinformatics analysis of common differential genes of coronary artery disease and ischemic cardiomyopathy. Eur Rev Med Pharmacol Sci 2018;22:3553-69.

16. Murphy CC, Wallace K, Sandler RS, et al. Racial disparities in incidence of young-onset colorectal cancer and patient survival. Gastroenterology 2019;156:958-65.

17. Dekker E, Rex DK. Advances in CRC prevention: screening and surveillance. Gastroenterology 2018;154:1970-84.

18. Vedeld HM, Goel A, Lind GE. Epigenetic biomarkers in gastrointestinal cancers: the current state and clinical perspectives. Semin Cancer Biol 2018;51:36-49.

19. Sveen A, Kopetz S, Lothe RA. Biomarker-guided therapy for colorectal cancer: strength in complexity. Nat Rev Clin Oncol 2020;17:11-32.

20. Ciardiello D, Vitiello PP, Cardone C, et al. Immunotherapy of colorectal cancer: Challenges for therapeutic efficacy. Cancer Treat Rev 2019;76:22-32.

21. Tang Z, Li C, Kang B, et al. GEPIA: a web server for cancer and normal gene expression profiling and interactive analyses. Nucleic Acids Res 2017;45:W98-102. 
22. Kaizuka T, Hara T, Oshiro N, et al. Ttil and Tel2 are critical factors in mammalian target of rapamycin complex assembly. J Biol Chem 2010;285:20109-16.

23. Sugimoto K. Branching the Tel2 pathway for exact fit on phosphatidylinositol 3-kinase-related kinases. Curr Genet 2018;64:965-70.

24. Takai H, Xie Y, de Lange T, et al. Tel2 structure and function in the Hsp90-dependent maturation of mTOR and ATR complexes. Genes Dev 2010;24:2019-30.

25. Garcia N, Li Y, Dooner HK, et al. Maize defective kernel mutant generated by insertion of a Ds element in a gene encoding a highly conserved TTI2 cochaperone. Proc Natl Acad Sci U S A 2017;114:5165-70.

26. Ma Z, Lou S, Jiang Z. PHLDA2 regulates EMT and autophagy in colorectal cancer via the PI3K/AKT signaling pathway. Aging (Albany NY) 2020;12:7985-8000.

27. Shaik A, Kirubakaran S. Evolution of PIKK family kinase inhibitors: a new age cancer therapeutics. Front Biosci (Landmark Ed) 2020;25:1510-37.

28. Strzeszewska A, Alster O, Mosieniak G, et al. Insight into the role of PIKK family members and NF-кB in DNA damage-induced senescence and senescence-associated secretory phenotype of colon cancer cells. Cell Death Dis 2018;9:44.

29. Kim SG, Hoffman GR, Poulogiannis G, et al. Metabolic stress controls mTORC1 lysosomal localization and dimerization by regulating the TTT-RUVBL1/2 complex. Mol Cell 2013;49:172-85.

30. Hurov KE, Cotta-Ramusino C, Elledge SJ. A genetic screen identifies the Triple $\mathrm{T}$ complex required for DNA

Cite this article as: $\mathrm{Xu}$ P, Du G, Guan H, Xiao W, Sun L, Yang H. A role of TTI1 in the colorectal cancer by promoting proliferation. Transl Cancer Res 2021;10(3):1378-1388. doi: $10.21037 /$ tcr-20-3322 damage signaling and ATM and ATR stability. Genes Dev 2010;24:1939-50.

31. Fernández-Sáiz V, Targosz B, Lemeer S, et al. SCFFbxo9 and CK2 direct the cellular response to growth factor withdrawal via Tel2/Tti1 degradation and promote survival in multiple myeloma. Nat Cell Biol 2013;15:72-81.

32. Cancer Genome Atlas Research Network. Comprehensive molecular characterization of urothelial bladder carcinoma. Nature 2014;507:315-22.

33. Rao F, Cha J, Xu J, et al. Inositol pyrophosphates mediate the DNA-PK/ATM-p53 cell death pathway by regulating CK2 phosphorylation of Tti1/Tel2. Mol Cell 2014;54:119-32.

34. Ni F, Tang H, Wang C, et al. Baohuoside I inhibits the proliferation of pancreatic cancer cells via mTOR/S6K1Caspases/Bcl2/Bax apoptotic signaling. Cancer Manag Res 2019;11:10609-21.

35. Sun X, Kaufman PD. Ki-67: more than a proliferation marker. Chromosoma 2018;127:175-86.

36. Zhou H, Huang T, Xiong Y, et al. The prognostic value of proliferating cell nuclear antigen expression in colorectal cancer: a meta-analysis. Medicine (Baltimore) 2018;97:e13752.

37. Hanahan D, Weinberg RA. Hallmarks of cancer: the next generation. Cell 2011;144:646-74.

38. Lee N, Kim D. Cancer metabolism: fueling more than just growth. Mol Cells 2016;39:847-54.

39. El Hout M, Cosialls E, Mehrpour M, et al. Crosstalk between autophagy and metabolic regulation of cancer stem cells. Mol Cancer 2020;19:27. 

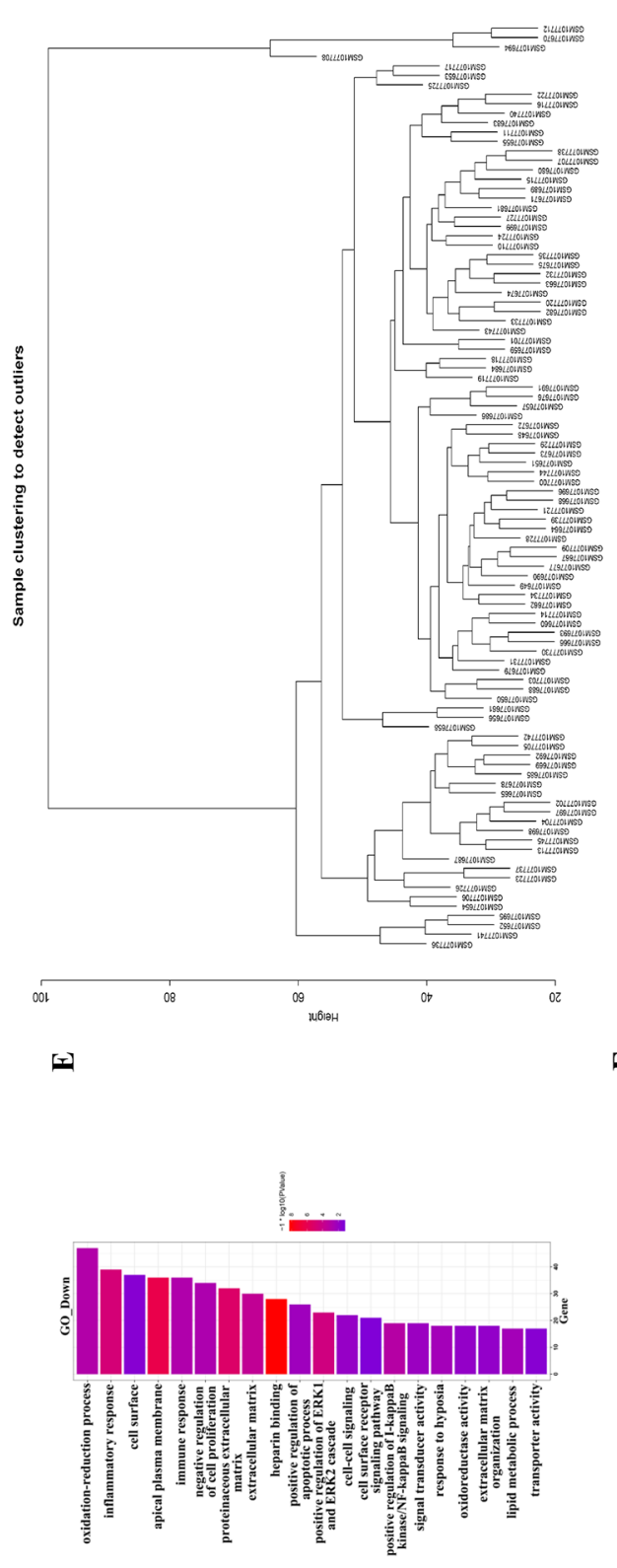

$\infty$

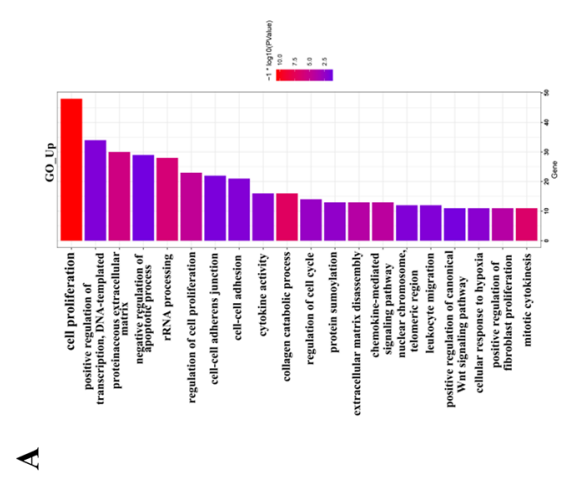

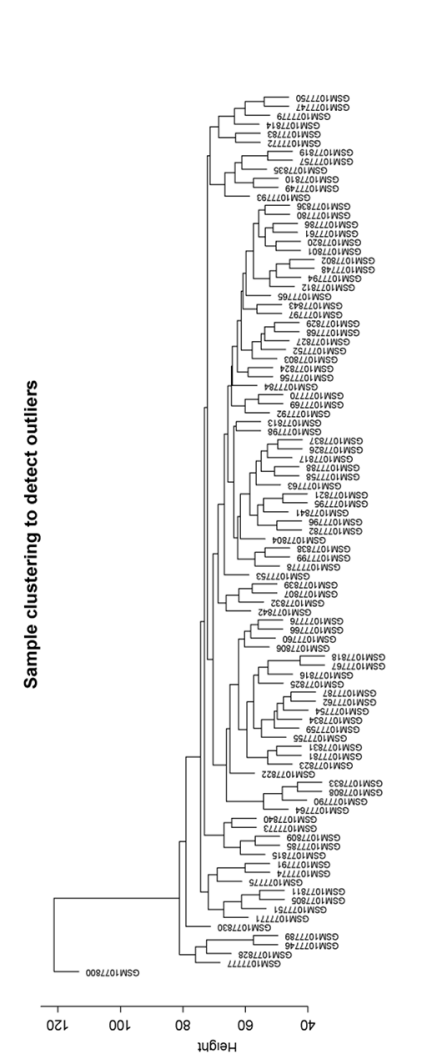
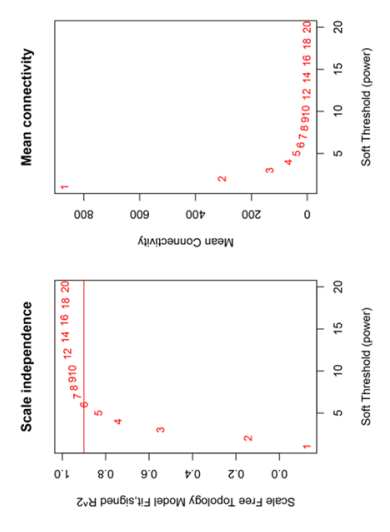

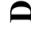
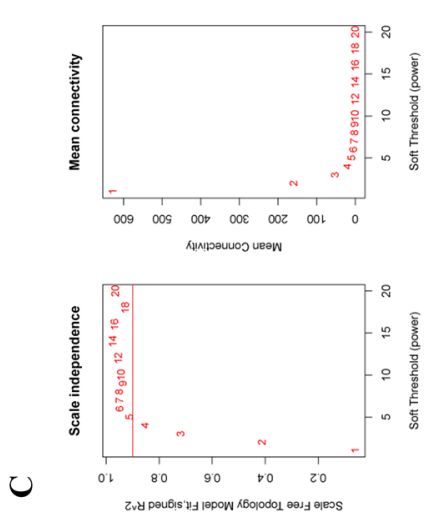

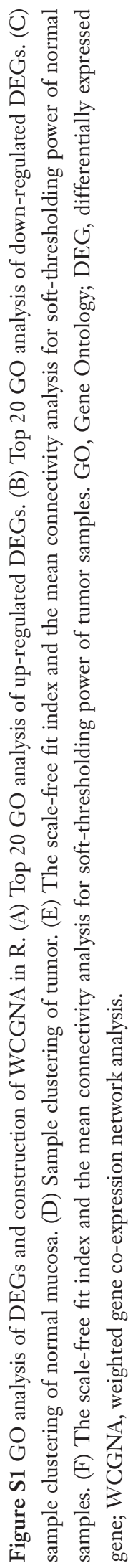

(C) Translational Cancer Research. All rights reserved. 


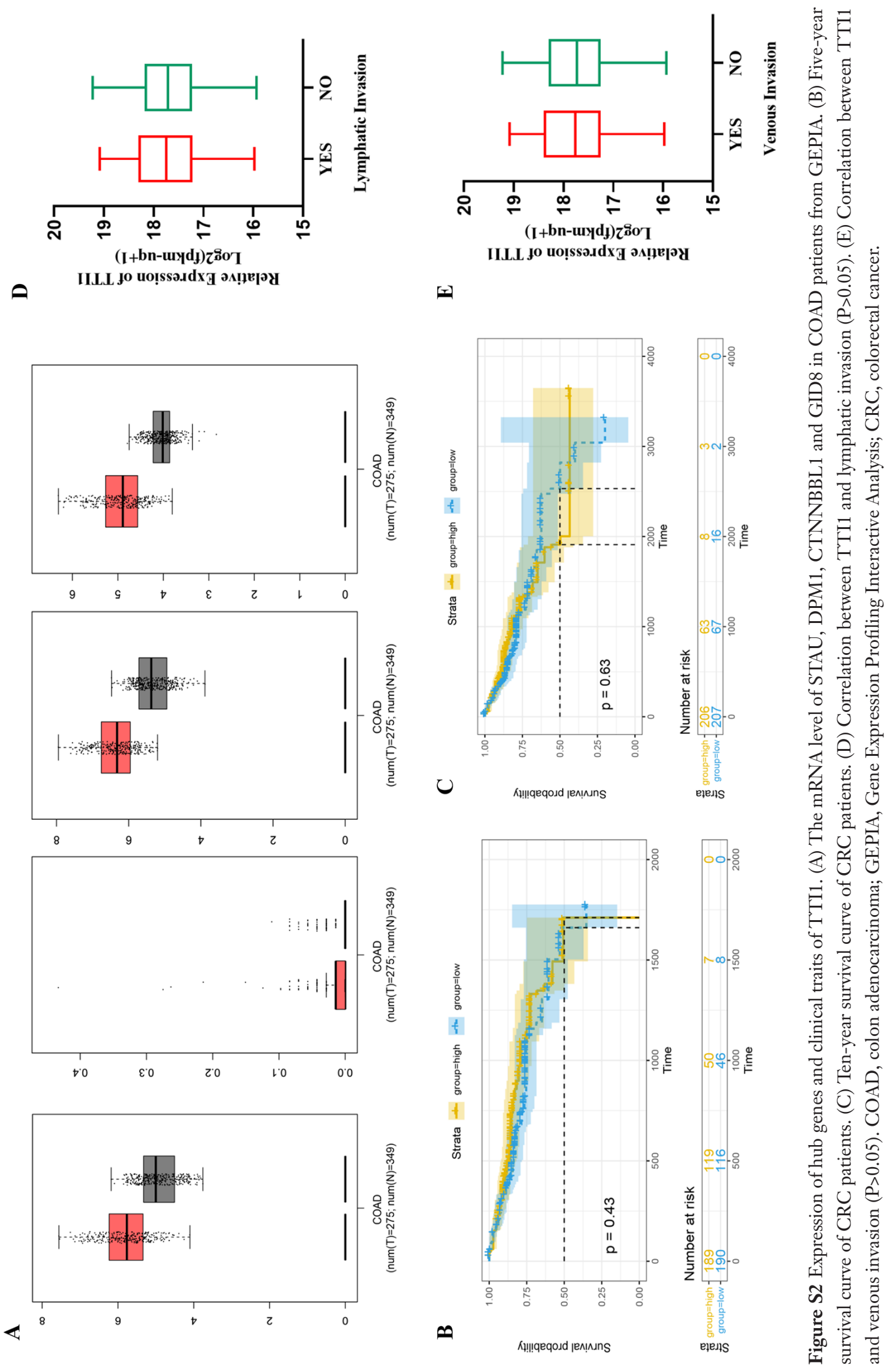

